\begin{tabular}{|l|l|}
\hline $\begin{array}{l}\text { 2. To: (Receiving organization) } \\
\text { Distribution }\end{array}$ & $\begin{array}{l}\text { 3. From: (Originating organization) } \\
\text { Sesimic Monitoring }\end{array}$ \\
\hline 5. Proj./Prog./Dept./0iv.: & 6. Cog. Engr.: \\
Seismic Monitoring & D.C. Hartshorn Sma \\
& S.P. Reidel
\end{tabular}

8. Originator Remarks:

Earthquakes recorded at and near Hanford between April 1, 1996 and June 30, 1996.
4. Related EDT No.:

NA

7. Purchase Order No.:

NA

9. Equip./Component No.:

NA

10. System/Bldg./Facility:

NA

\section{Receiver Remarks:}

12. Major Assm. Dwg. Mo.:

NA

13. Permit/Permit Application Mo.: NA

14. Required Response Date:

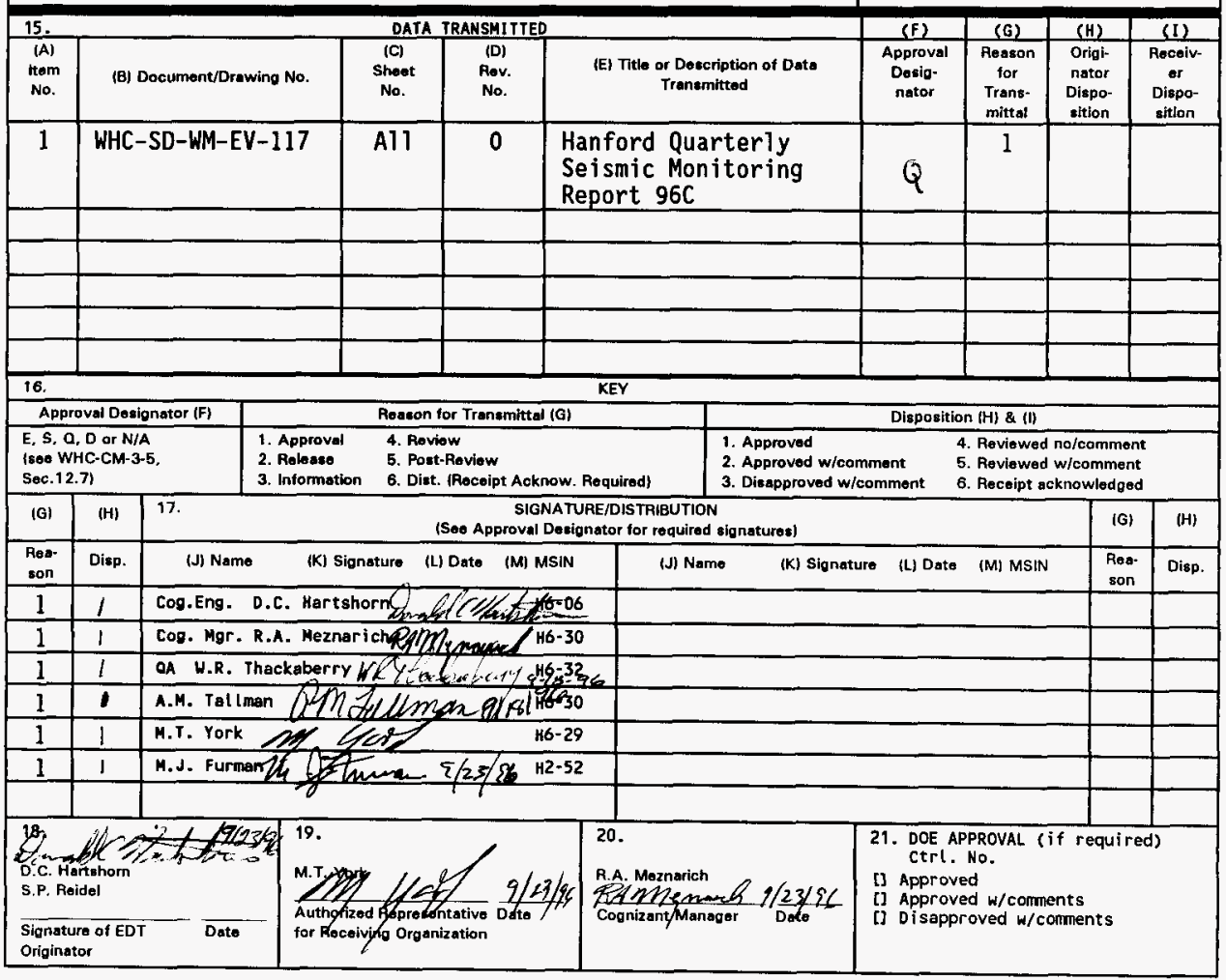

8D-7400-172-2 (04/94) GEF097 


\title{
Quarterly Seismic Monitoring Report 96C
}

\author{
D.C. Hartshorn
}

S.P. Reidel

Westinghouse Hanford Co., Richland, WA 99352

U.S. Department of Energy Contract DE-AC06-87RL10930
EDT/ECN: 610760
UC: 903
Org Code: $8 \mathrm{H} 200$
Charge Code: R4VA3
B\&R Code: EW3120100
Total Pages: 28

Key Words: Seismicity, Sesimic Monitoring, Earthquakes, Tectonics

Abstract: This report summarizes the location, magnitude, and other pertinent information on earthquakes recorded on and near the Hanford Site by Westinghouse Seismic Monitoring during the period encompassing April 1, 1996 to June 30, 1996. TRAOEMARK OISCLAIMER. Reference herein to any specific comercial product, process, or service by
trade name, trademark, manufacturer, or otherwise, does not necessarily constitute or imply its
endorsement, recoamendation, or favoring by the United States Government or any agency thereof or its contractors or subcontractors.

Printed in the United states of America. To obtain copies of this document, contact: WHC/BCS Document Control Services, P.0. Box 1970, Mailstop H6-08, Richland WA 99352, Phone (509) 372-2420: Fax (509) 376-4989.

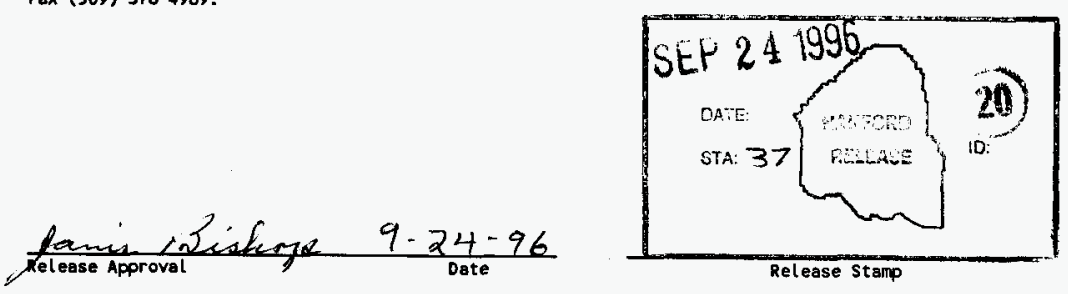

Approved for Public Release 
WHC-SD-WM-EV-117, Rev. 0

This page intentionally left blank. 


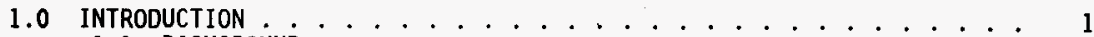

1.1 BACKGROUND . . . . . . . . . . . . . . . . . . . . . . 1

1.3 REPORTS . . . . . . . . . . . . . . . . . . . . 1

2.0 NETWORK OPERATIONS . . . . . . . . . . . . . . . . . . . . . . 2

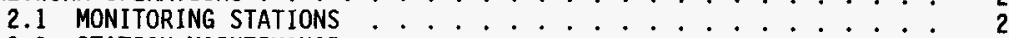

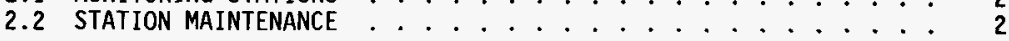

3.0 EXPLANATION OF MAGNITUDE AND VELOCITY MODEL . . . . . . . . . . . . . . 8

3.1 CODA AMPLITUDE MAGNITUDE ..................... . . . . 8

3.2 VELOCITY MODEL . . . . . . . . . . . . . . . . . . . . . . . 8

4.0 GEOLOGY AND TECTONIC ANALYSIS . . . . . . . . . . . . . . . . . . 13

4.1 DEPTH OF EARTHQUAKES ....................... . . . . . . 13

4.2 TECTONIC PATTERN . . . . . . . . . . . . . . . . . . . . . 13

4.3 CURRENT TECTONIC ACTIVITY . . . . . . . . . . . . . . . . . . . 14

4.3.1 Reverse/Thrust Faults on Major Anticlinal Ridges . . 18

4.3.2 Secondary Structures on Main Anticlinal Ridges . . . . 18

4.3.3 Swarm Area Activity .. . . . . . . . . . . . . . 18

4.3.4 Random or Floating Event Areas . . . . . . . . . . . 19

5.0 REFERENCES ......................... 20 


\section{LIST OF FIGURES}

1 Locations of Seismograph Stations ............... 4

2 Locations of Seismograph Stations in the Eastern Washington Regional Network ................. 6

3 A11 Located Earthquakes: Apri1 1, 1996, and June $30,1996 \ldots \ldots 16$

4 Locations of All FY 1996 Events: . . . . . . . . . . . . 17

5 Structural and Tectonic Map of Columbia Basin Showing Major Seismic Source Structures . . . . . . . . . . . . . 18

\section{LIST OF TABLES}

I Stations Used for Location of Events ............. . . 3

2 Station Locations for the Eastern Washington Regional Network $\ldots . .5$

3 Seismic Channel Operational Time .............. 7

4 Velocity Model for the Columbia Basin ............... 9

5 Seismic Event Data ................... 10 
WHC-SD-WM-EV-117, Rev. 0

\section{TERMS}

BWIP

CAM

CRBG

CUT

DOE

EWRN

FY

GBB

GPS

HSN

HTS

NEL

RAW

$\mathrm{RC} 1$

SMART

USGS

WHC
Basalt Waste Isolation Project

Coda Amplitude Magnitude

Columbia River Basalt Group

Coordinated Universal Time

U.S. Department of Energy

Eastern Washington Regional Network

fiscal year

Gable Butte

Global Positions Satellite System

Hanford Seismic Network

Hanford Technical Services

Nelson Butte

Rattlesnake Mountain-Wallula Alignment

Royal City

Seismic Monitoring Analysis and Repair Team

United States Geological Survey

Westinghouse Hanford Company 
WHC-SD-WM-EV-117, Rev. 0

This page intentionally left blank. 
WHC-SD-WM-EV-117, Rev. 0

\subsection{INTRODUCTION}

\subsection{BACKGROUND}

Seismic monitoring at the Hanford Site was established in 1969 by the United States Geological Survey (USGS) under a contract with the U.S. Atomic Energy Commission. In 1975 the University of Washington assumed responsibility for and expanded the network. In 1979 the Basalt Waste Isolation Program (BWIP) became responsible for collecting seismic data for the site as part of site characterization. Rockwell International Operations followed by Westinghouse Hanford Company (WHC), Geosciences Group, operated the local network and were the contract technical advisors for the Eastern Washington Regional Network operated by the University of Washington. Funding ended for BWIP in December 1988. Seismic Monitoring and the University of Washington contract was then transferred WHC's Environmental Division.

Seismic Monitoring is currently assigned to WHC's Hanford Technical Services (HTS), part of the Environmental Division. The Seismic Monitoring Analysis and Repair Team (SMART) operates, maintains, and analyzes data from the Hanford Seismic Network (HSN), extending the site historical seismic database and fulfilling U.S. Department of Energy, Richland Operations Office requirements and orders. The Seismic Monitoring Analysis and Repair Team also maintains the Eastern Washington Regional Network (EWRN). The University of Washington uses the data from the EWRN and other seismic networks in the Northwest to provide the SMART with necessary regional input for the seismic hazards analysis at the Hanford Site.

\subsection{PURPOSE}

The SMART is tasked to provide an uninterrupted collection of high-quality raw seismic data from the HSN located on and encircling the Hanford Site. These unprocessed data are permanently archived. The SMART is also tasked to locate and identify sources of seismic activity, monitor changes in the historical pattern of seismic activity at the Hanford Site, and build a "local" earthquake database (processed data). Local earthquakes are defined for this report and database as earthquakes that occur within 46 degrees to 47 degrees West Longitude and 119 degrees to 120 degrees North Latitude. The data are used by the SMART, Waste Management, and engineering activities at the Hanford Site to evaluate seismic hazards and seismic design for the Site. The processed database is also permanently archived.

\subsection{REPORTS}

The SMART issues brief monthly activity reports, quarterly reports with discussion of local activity, an annual catalog of earthquake activity on and near the Site, and bulletins on special-interest local seismic events. The SMART also provides information and reports to other functions, as well as educational assistance to area schools and programs when the assistance is requested and approved, and the workload permits. 
WHC-SD-WM-EV-117, Rev. 0

\subsection{NETWORK OPERATIONS}

\subsection{MONITORING STATIONS}

The HSN and the EWRN have 40 individual sensor sites. Sixteen sites are shared by both networks, so that the Hanford Seismic Network has 21 sites (Table 1 and Figure 1) and the Eastern Washington Regional Network has 35 (Table 2 and Figure 2). The networks have 44 combined data channels because Gable Butte and Royal City are three-component sites, each consisting of one vertical, one north-south horizontal, and one east-west horizontal data channe1. Both networks use 15 additional telemetry relay sites. All station data links pass through at least one of these relay sites. Some data links cross as many as three relay sites. The last section of the data link for all channels is commercial telephone lines. Three to eight channels are multiplexed per telephone line.

Most stations and five relay sites are powered by solar panels and batteries. A major outage is considered to be a failure of the HSN data recording computer or three or more data channels.

\subsection{STATION MAINTENANCE}

The HSN's station outages and a review of the station maintenance for the current quarter can be found in the Seismic Monitoring monthly reports. Table 3 shows the percentage of time the channel was operational for the period. One site in the EWRN, Nelson Butte (NEL), is currently inoperative. Access to repair NEL is prevented by damaged roads and the site's remote location. The site is located in the northern Cascades at 1,490 m elevation. A repair trip is scheduled for late August 1996. 
Table 1. Stations Used for Location of Events.

\begin{tabular}{|c|c|c|c|c|}
\hline Station & $\begin{array}{l}\text { Lat i tude } \\
\text { Deg.Min.N }\end{array}$ & $\begin{array}{l}\text { Longi tude } \\
\text { Deg.Min.W }\end{array}$ & Elevation (m) & Station Nane \\
\hline BEN & $46-31.13$ & $119-43.02$ & 340 & Benson Ranch \\
\hline BRV & $46-49.12$ & $119-59.47$ & 920 & Black Rock Valley \\
\hline BVW & $46-48.66$ & $119-52.99$ & 670 & Beverly, Washington \\
\hline CRF & $46-49.50$ & $119-23.22$ & 189 & Corfu, Washington \\
\hline ET3 & $46-34.64$ & $118-56.25$ & 286 & El topia Three \\
\hline *GBB & $46-36.49$ & $119-37.62$ & 177 & Gable Butte \\
\hline GBL & $46-35.92$ & $119-27.58$ & 330 & Gable Mountain \\
\hline $\mathrm{H} 2 \mathrm{O}$ & $46-23.75$ & $119-25.38$ & 158 & Woter \\
\hline LOC & $46-43.02$ & $119-25.85$ & 210 & Locke is land \\
\hline MOW & $46-36.79$ & $119-45.66$ & 330 & Midway, Washington \\
\hline$M \sqrt{2}$ & $46-33.45$ & $119-21.54$ & 146 & May Junction Two \\
\hline OI3 & $46-40.14$ & $119-13.98$ & 322 & Othel lo Three \\
\hline PRO & $46-12.73$ & $119-41.15$ & 550 & Prosser, Hashington \\
\hline *RC1 & $46-56.71$ & $119-26.66$ & 485 & Royal city One \\
\hline REO & $46-17.92$ & $119-26.30$ & 366 & Red Mountain \\
\hline RSW & $46-23.67$ & $119-35.48$ & 1,045 & Rattlesnake Mt. WA \\
\hline SNI & $46-27.85$ & $119-39.60$ & 312 & Snively Ranch \\
\hline WA2 & $46-45.32$ & $119-33.94$ & 244 & Wahluke slope \\
\hline WG4 & $46-01.85$ & $118-51.34$ & 511 & Wal lula Gap Four \\
\hline HIH & $46-25.76$ & $119-17.26$ & 128 & Wooded is L and WA \\
\hline URD & $46-58.20$ & $119-08.69$ & 375 & Warden, Washington \\
\hline
\end{tabular}


Figure 1. Locations of Seismograph Stations.

(See Table 1 for exact locations.)

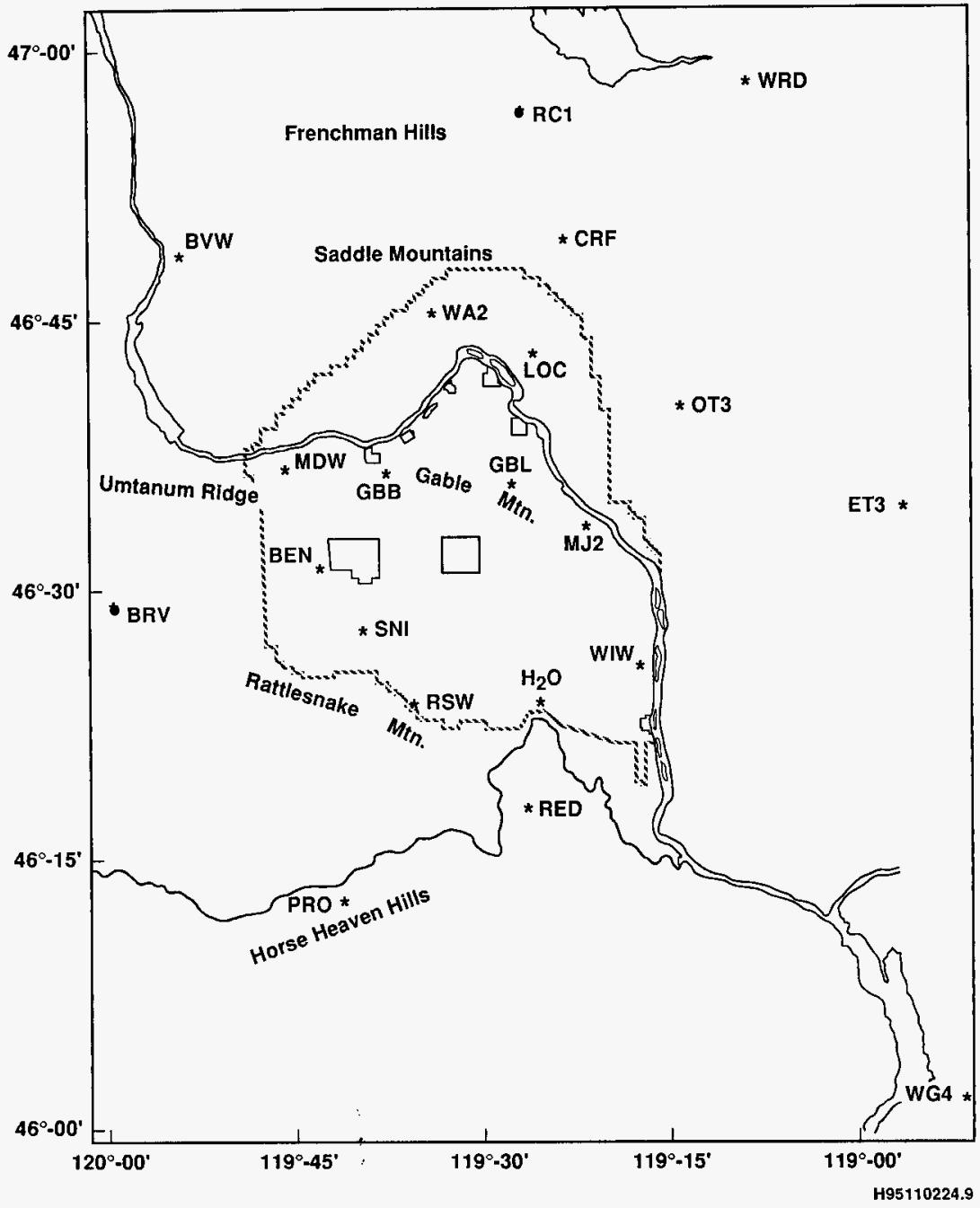


Table 2. Station Locations for the Eastern Washington Regional Network. This table lists stations of the Eastern Washington Regional Network. The first colum is the threeletter seismic station designator. This is followed by the latitude-north in degrees, minutes, and hundredths of minutes; the longitude-west in degrees, minutes, and hundredths of minutes; elevation above sea level in meters; and the full station name. An asterisk before the three-letter designator means it is a three-component station. The locations of the stations were derived from a Global Position Satellite system (GPS).

\begin{tabular}{|c|c|c|c|c|}
\hline Station & $\begin{array}{l}\text { Lat i tude } \\
\text { Deg.Min.N. }\end{array}$ & $\begin{array}{l}\text { Long i tude } \\
\text { Deg.Min.W. }\end{array}$ & Elevation (m) & Station Name \\
\hline BRV & $46-29.12$ & $119-59.47$ & 920 & Black Rock Val ley \\
\hline BVI & $46-48.66$ & $119-52.99$ & 670 & Beverly Wash ington \\
\hline CBS & $47-48.26$ & $120-02.50$ & 1,067 & Chelan Butte, South \\
\hline CRF & $46-49.50$ & $119-23.22$ & 189 & Corfu \\
\hline DPH & $47-52.25$ & $118-12.17$ & 892 & Davenport Washington \\
\hline DY2 & $47-59.11$ & $119-46.28$ & 890 & Dyer Hill Two \\
\hline Ell & $46-54.58$ & $120-33.98$ & 789 & Ellensburg \\
\hline EPH & $47-21.38$ & $119-35.76$ & 661 & Ephrata \\
\hline ET3 & $46-34.64$ & $118-56.25$ & 286 & El topia Three \\
\hline ETW & $47-36.26$ & $120-19.94$ & 1,477 & Entiat Washington \\
\hline GBL & $46-35.92$ & $119-27.58$ & 330 & Gable Mountain \\
\hline LNO & $45-52.31$ & $118-17.11$ & 771 & Lincton $\mathrm{Mt}_{\mathrm{t}}$ Oregon \\
\hline LOC & $46-43.02$ & $119-25.85$ & 210 & Locke Island \\
\hline MDH & $46-36.79$ & $119-45.66$ & 330 & Midway Washington \\
\hline Md2 & $46-33.45$ & $119-21.54$ & 146 & May Junction Two \\
\hline $\operatorname{mox}$ & $46-34.64$ & $120-17.89$ & 501 & Moxee City \\
\hline MAC & $46-43.99$ & $120-49.42$ & 728 & Naches \\
\hline MEL & $48-04.21$ & $120-20.41$ & 1,500 & Nel son Butte \\
\hline 002 & $47-23.26$ & $118-42.58$ & 553 & Odessa Two \\
\hline 013 & $46-40.14$ & $119-13.98$ & 322 & Dthello Three \\
\hline PAT & $45-52.92$ & $119-45.14$ & 262 & Paterson \\
\hline PRO & $46 \cdot 12.73$ & $119-41.15$ & 550 & Prosser \\
\hline *RC1 & $46-56.71$ & $119-26.66$ & 485 & Royal City One \\
\hline RSW & $46-23.67$ & $119-35.48$ & 1,045 & Rattlesnake Mt. WA \\
\hline SAN & $47-42.10$ & $119-24.03$ & 701 & St. Andrews \\
\hline SNI & $46-27.85$ & $119-39.60$ & 312 & Snively Ranch \\
\hline TEM & $47-10.20$ & $120-35.88$ & 1,006 & Table Mountain \\
\hline THW & $47-08.29$ & $120-52.10$ & 1,027 & Teanaway Washington \\
\hline VT2 & $46-58.04$ & $119-58.95$ & 1,270 & Vantage Two \\
\hline WA2 & $46-45.32$ & $119-33.94$ & 244 & Wahluke slope Two \\
\hline WAT & $47-41.92$ & $119-57.24$ & 821 & Waterville \\
\hline HG4 & $46-01.85$ & $118-51.34$ & 511 & Wallula Gap four \\
\hline WIW & $46-25.76$ & $119-17.26$ & 128 & Wooded Is land WA \\
\hline URD & $46-58.20$ & $119-08.69$ & 375 & Warden \\
\hline YA2 & $46-31.60$ & $120-31.80$ & 652 & Yakima Two \\
\hline
\end{tabular}


Figure 2. Locations of Seismograph Stations in the Eastern Washington Regional Network. (See Table 2 for exact locations.)

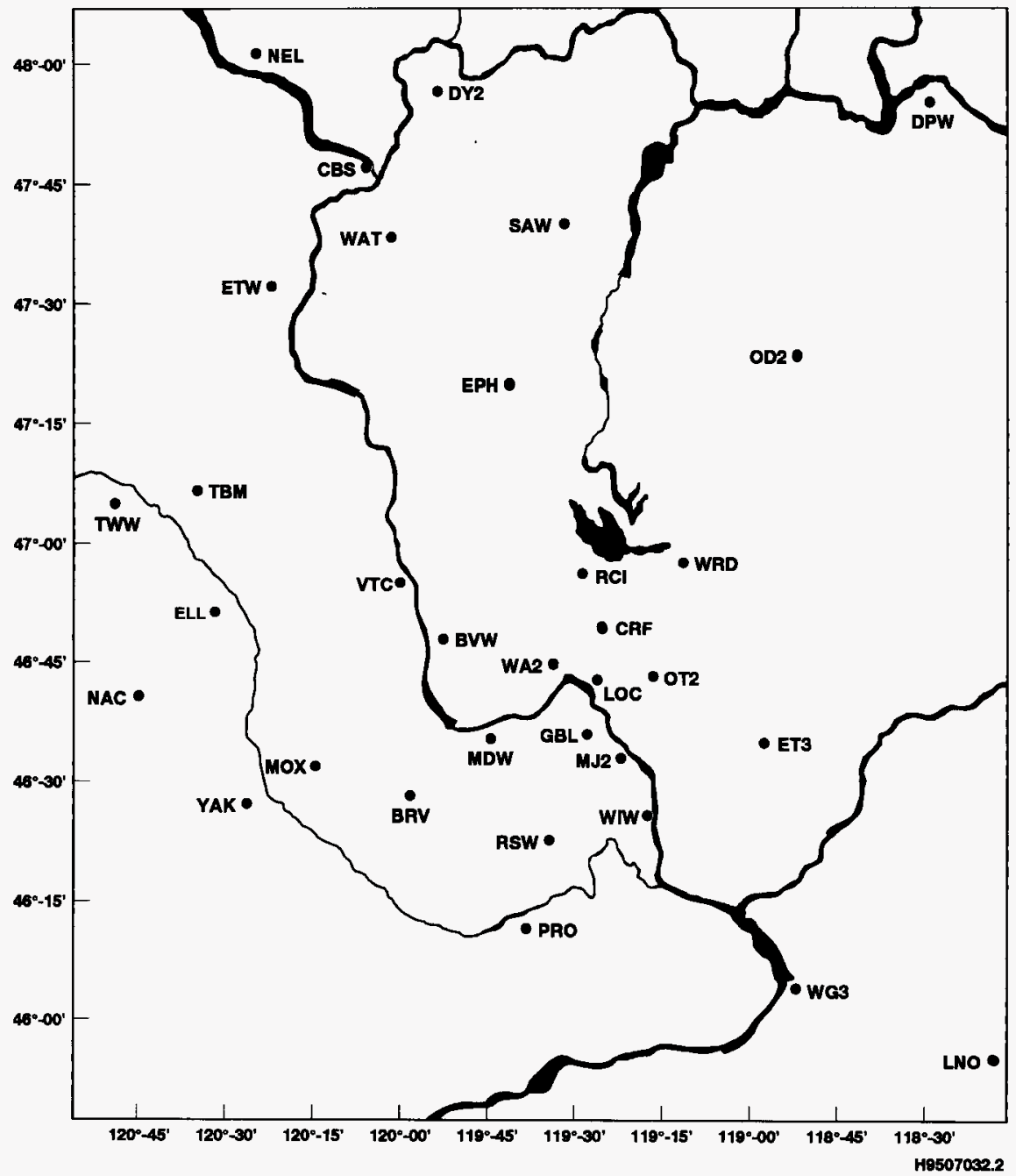


Table 3. Seismic Channel Operational Time (\%).

\begin{tabular}{|l|c|c|c|c|c|}
\hline \multicolumn{1}{|c|}{ Network } & April & May & June & 2nd Quarter & FY-Year \\
\hline Hanford & 99.8 & 100.0 & 99.9 & 99.9 & 99.8 \\
\hline Regional & 97.2 & 97.3 & 97.3 & 97.3 & 98.6 \\
\hline
\end{tabular}

This column is the average percentage for the second quarter.

'This column is the average percentage for fiscal year 1996.

Notes: The Hanford Seismic Network's goal is $98 \%$.

The Eastern Washington Regional Network's goal is $97 \%$.

The quarter period is April 1, 1996 through June 30, 1996. 


\subsection{EXPLANATION OF MAGNITUDE AND VELOCITY MODEL}

A11 magnitudes given in this report are Coda Amplitude Magnitudes (CAM). CAMs are the equivalent of Richter Magnitudes and apply only to local earthquakes.

\subsection{CODA AMPLITUDE MAGNITUDE}

The AUTOSEIS program calculates the location, depth, and magnitude for earthquakes detected at the Hanford Site. This program uses the basic decay of the $S$ wave. By quantifying the expected decay of the average absolute amplitude as a function of time, any portion of the coda of an earthquake (coda segment) can be used to assign a magnitude. For the high-frequency earthquakes analyzed at the Hanford Site, the coda segment length is a 1 -second sample. A number of coda segments of each record can be averaged (or a median value selected) to produce a robust estimate of magnitude for each station. The average magnitudes for all stations are then averaged for an assigned CAM for that particular earthquake.

The CAM for each segment is computed from the following:

$$
C A M=\log A+Q \log (t-B)
$$

where:

$$
A=1 / 256 \sum_{i=1}^{256}\left|S_{i}\right|
$$

$S_{i}=$ signal amplitude at sample

$t=$ signal coda window in seconds $=1.0$

$Q$ describes the decay rate of the signal and $B$ describes any DC bias for a particular station. Both parameters are station dependent.

\subsection{VELOCITY MODEL}

The velocities and layer depths presented in Table 4 are used by the AUTOSEIS program to locate all earthquakes on and near the Hanford Site. 
WHC-SD-WM-EV-117, Rev. 0

Table 4. Velocity Model for the Columbia Basin.

\begin{tabular}{|c|l|c|}
\hline $\begin{array}{c}\text { Depth to top of } \\
\text { velocity layer }(\mathrm{km})\end{array}$ & \multicolumn{1}{|c|}{ Stratigraphy } & $\begin{array}{c}\text { Velocity } \\
(\mathrm{km} / \mathrm{sec})\end{array}$ \\
\hline 0.0 & $\begin{array}{l}\text { Suprabasalt sediments, Saddle Mountains } \\
\text { Basalt and intercalated El lensburg Sediments }\end{array}$ & 3.7 \\
\hline 0.8 & Wanapum Basalt & 4.7 \\
\hline 1.2 & Grande Ronde Basalt & 5.2 \\
\hline 7.5 & Prebasalt Sediments & 6.1 \\
\hline 19.0 & Crystalline Basement, Layer 1 & 7.2 \\
\hline 28.0 & Crystalline Basement, Layer 2 & 8.0 \\
\hline
\end{tabular}


Table 5. Seismic Event Data. (3 sheets)

Event ID: The identification number assigned by the AUTOSEIS program

Date: $\quad$ The day of the year in Coordinated Universal Time (CUT).

Time: The origin time of the earthquake given in CUT. To convert CUT to Pacific Standard Time, subtract eight hours; to Pacific Daylight Time, subtract seven hours.

Latitude: North latitude, in degrees and minutes, of the earthquake epicenter.

Longitude: West longitude, in degrees and minutes, of the earthquake epicenter.

Depth: $\quad$ The depth of the earthquake in kilometers $(\mathrm{km})$.

Mag: The Coda Amplitude Magnitude (CAM). The CAM is calculated using an algorithm modified for very local earthquakes: AUTOSEIS Reference Manual, October 20, 1983, Sierra Geophysics, Inc. (SGI-M-AS-0038). See Page 5 of this report: Explanation of Magnitude and Velocity Model.

RMS: The root mean square residual (observed P-WAVE arrival time minus the predicted arrival time) at all stations used to locate the earthquake. It is only useful as a measure of quality of the solution when five or more well distributed stations are used in the solution. Good solutions are normally characterized by RMS values of less than about 0.3 second.

Q: $\quad$ The solution quality of the hypocenter intended to indicate the general reliability of the solution: AUTOSEIS Reference Manual, October 20, 1983, Sierra Geophysics Inc. (SGI-M-AS-0038). The quality is rated using characters $A, B, C$, and D.

\begin{tabular}{|c|c|c|c|}
\hline$Q$ & Error $(\mathrm{km})$ & Epicenter & Focal Depth \\
\hline & $\leq 1.0$ & Excellent & Good \\
\hline & $\leq 2.5$ & Good & Fair \\
\hline & $\leq 5.0$ & Fair & Poor \\
\hline$D$ & $>5.0$ & Poor & Poor \\
\hline
\end{tabular}

Events that occur at the edge or outside of the Hanford Seismic Network or are detected by fewer than five stations very often have a $Q$ of $C$ or $D$.

*Denotes events that occurred during this quarter. 
WHC-SD-WM-EV-117, Rev. 0

\begin{tabular}{|c|c|c|c|c|c|c|c|c|c|c|c|c|c|c|c|c|c|c|}
\hline 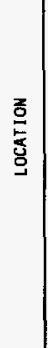 & 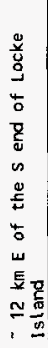 & 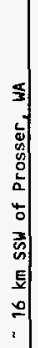 & 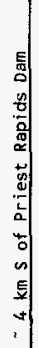 & 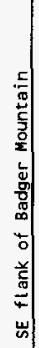 & 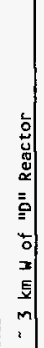 & 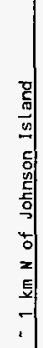 & 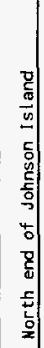 & 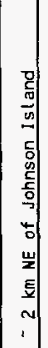 & 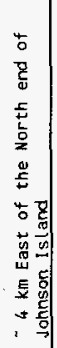 & 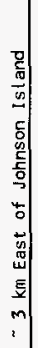 & 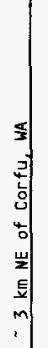 & 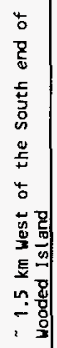 & 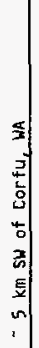 & 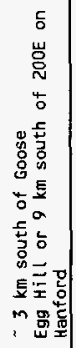 & 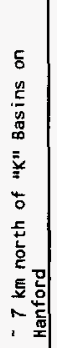 & 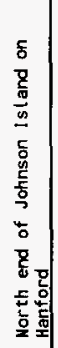 & 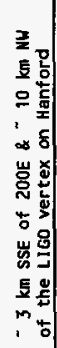 & 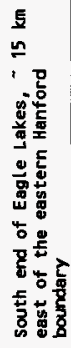 \\
\hline 0 & $\infty$ & $\omega$ & 4 & 0 & $\infty$ & $\infty$ & $\infty$ & $\infty$ & $\infty$ & 0 & 4 & $<$ & $\infty$ & $\alpha$ & $\infty$ & m & $<$ & $\infty$ \\
\hline 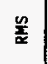 & $\begin{array}{l}\text { us } \\
\dot{0}\end{array}$ & 古 & $\begin{array}{l}\text { m. } \\
0 \\
0\end{array}$ & 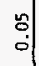 & $\begin{array}{l}0 \\
0 \\
0\end{array}$ & $\begin{array}{l}\text { tे } \\
\text { वे. }\end{array}$ & : & 氙 & $\begin{array}{l}\text { Jे } \\
\dot{\sigma}\end{array}$ & $\begin{array}{l}\text { : } \\
0 \\
0\end{array}$ & $\begin{array}{l}\text { 告 } \\
0\end{array}$ & $\begin{array}{l}\text { oे } \\
0\end{array}$ & مै: & 옹 & 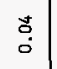 & $\stackrel{\circ}{\circ}$ & $\begin{array}{l}\text { U. } \\
\text { ○' }\end{array}$ & $\begin{array}{l}\text { d̆ } \\
\dot{d}\end{array}$ \\
\hline$\frac{\pi}{2}$ & ํ. & $=$ & $\stackrel{\simeq}{-}$ & $\stackrel{\infty}{=}$ & $\tilde{n}$ & ले & $\begin{array}{l}n \\
- \\
-\end{array}$ & $\stackrel{a}{=}$ & ? & $\stackrel{-}{\therefore}$ & 号 & $\frac{a}{i}$ & $\begin{array}{l}8 \\
8 \\
0\end{array}$ & 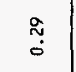 & $\cong$ & $\stackrel{R}{R}$ & مa & 范 \\
\hline 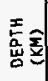 & $\begin{array}{l}\stackrel{8}{\circ} \\
\stackrel{\sim}{\sim}\end{array}$ & 열 & $\cong$ & $\begin{array}{l}0 \\
\stackrel{0}{0} \\
\stackrel{0}{2}\end{array}$ & $\stackrel{\infty}{\circ}$ & $\begin{array}{l}0 \\
0 \\
0 \\
0\end{array}$ & $\begin{array}{l}0 \\
\vdots \\
\vdots\end{array}$ & : & $\stackrel{\infty}{\sim}$ & $\begin{array}{l}0 \\
0 \\
0\end{array}$ & $\approx$ & $\underset{\sim}{\stackrel{d}{\sim}}$ & $\cong$ & 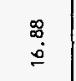 & $\stackrel{\circ}{\check{\sigma}}$ & $\begin{array}{l}\mathscr{0} \\
\vdots \\
0\end{array}$ & $\underset{\sim}{\tilde{N}}$ & $\frac{\stackrel{m}{m}}{m}$ \\
\hline 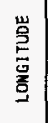 & $\begin{array}{l}\dot{0} \\
\infty \\
\frac{0}{1} \\
\frac{\alpha}{=}\end{array}$ & $\begin{array}{l}\overline{0} \\
a \\
a \\
a \\
=\end{array}$ & 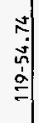 & $\begin{array}{l}\bar{n} \\
\frac{\infty}{\dot{\alpha}} \\
\underline{a}\end{array}$ & $\left|\begin{array}{c}a \\
\dot{\alpha} \\
\dot{a} \\
\frac{a}{q}\end{array}\right|$ & $\begin{array}{l}\alpha \\
\dot{a} \\
\underline{\alpha} \\
\dot{\alpha}\end{array}$ & 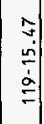 & 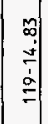 & 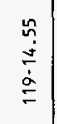 & $\begin{array}{l}\bar{\infty} \\
\dot{\vec{t}} \\
\overline{\mathbf{\alpha}} \\
\stackrel{\alpha}{=}\end{array}$ & $\begin{array}{l}\hat{y} \\
\hat{a} \\
\dot{a} \\
=\end{array}$ & $\begin{array}{l}\stackrel{2}{\vdots} \\
\frac{1}{\alpha} \\
\frac{1}{=}\end{array}$ & 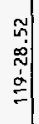 & 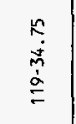 & $\begin{array}{l}= \\
\stackrel{a}{\alpha} \\
\stackrel{\alpha}{=}\end{array}$ & $\begin{array}{l}\text { M. } \\
\frac{0}{\dot{\alpha}} \\
\stackrel{\alpha}{=}\end{array}$ & $\begin{array}{l}\hat{n} \\
\stackrel{n}{n} \\
\stackrel{\alpha}{=} \\
=\end{array}$ & $\begin{array}{l}\text { ò } \\
\text { o } \\
\dot{\alpha} \\
\stackrel{\alpha}{=}\end{array}$ \\
\hline 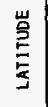 & 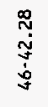 & 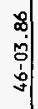 & $\begin{array}{l}- \\
0 \\
0 \\
0 \\
0 \\
0 \\
0\end{array}$ & $\begin{array}{c}m \\
\mathfrak{m} \\
\bar{c} \\
\vdots \\
q\end{array}$ & $\left|\begin{array}{c}0 \\
0 \\
y \\
y \\
0 \\
y\end{array}\right|$ & $\begin{array}{l}n \\
\\
\vdots \\
\vdots \\
0 \\
0\end{array}$ & 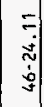 & $\begin{array}{l}a \\
\vdots \\
\vdots \\
\vdots \\
0\end{array}$ & 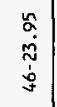 & 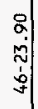 & $\begin{array}{l}\text { 영 } \\
0 \\
0 \\
0 \\
0\end{array}$ & 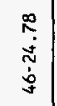 & $\begin{array}{l}0 \\
\vdots \\
\vdots \\
\dot{q} \\
\dot{y}\end{array}$ & $\begin{array}{l}n \\
\hat{n} \\
\vdots \\
0 \\
y\end{array}$ & 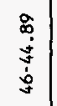 & 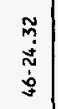 & $\begin{array}{l}\mathscr{g} \\
\circ \\
0 \\
\dot{p} \\
\dot{q}\end{array}$ & $\begin{array}{l}\text { m } \\
\text { g } \\
\vdots \\
\vdots \\
0\end{array}$ \\
\hline 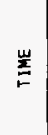 & 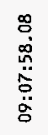 & 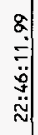 & 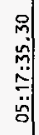 & $\begin{array}{c}0 \\
\vdots \\
: \\
\ddot{0} \\
\ddot{0} \\
\ddot{\vdots}\end{array}$ & 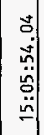 & $\begin{array}{l}\infty \\
0 \\
0 \\
\ddot{0} \\
\\
=\end{array}$ & 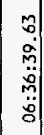 & $\left|\begin{array}{c}\infty \\
\ddot{0} \\
\ddot{a} \\
\ddot{n} \\
\ddot{a}\end{array}\right|$ & $\begin{array}{l}8 \\
\dot{-} \\
ي \\
\hat{j} \\
\ddot{~}\end{array}$ & 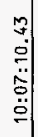 & $\begin{array}{l}0 \\
\ddot{0} \\
\ddot{0} \\
\ddot{0} \\
\ddot{0}\end{array}$ & 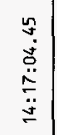 & 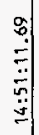 & $\begin{array}{l}\stackrel{m}{\circ} \\
\stackrel{\circ}{0} \\
\ddot{\circ} \\
\ddot{0}\end{array}$ & 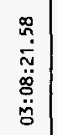 & 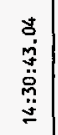 & 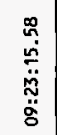 & 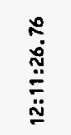 \\
\hline$\underset{\underline{\sigma}}{\underline{\sigma}}$ & $\frac{\pi}{0}$ & $\sum_{0}^{\infty}$ & 윙 & 号 & กับ & in & $\stackrel{\mathrm{m}}{\mathrm{n}}$ & 원 & 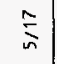 & $\stackrel{n}{n}$ & 昜 & $\sum_{j}^{\infty}$ & sู & ले & $\frac{\text { Nิ }}{\text { mิ }}$ & $\stackrel{m}{m}$ & $\stackrel{\bar{N}}{\mathrm{~m}}$ & 今 \\
\hline 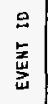 & 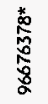 & 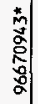 & 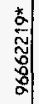 & 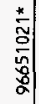 & 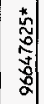 & $\left|\begin{array}{c}0 \\
0 \\
0 \\
0 \\
0 \\
0 \\
\vdots \\
0 \\
0\end{array}\right|$ & 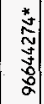 & $\begin{array}{l}\frac{*}{0} \\
\frac{\infty}{5} \\
\frac{0}{8}\end{array}$ & 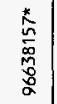 & 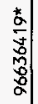 & 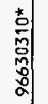 & 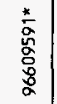 & $\begin{array}{l}\vdots \\
\vdots \\
\vdots \\
\vdots \\
\alpha \\
\alpha\end{array}$ & 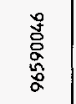 & 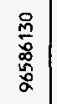 & 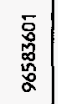 & 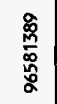 & 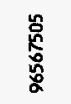 \\
\hline
\end{tabular}


WHC-SD-WM-EV-117, Rev. 0

\begin{tabular}{|c|c|c|c|c|c|c|c|c|c|c|c|c|c|c|c|c|}
\hline 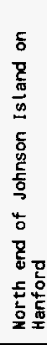 & 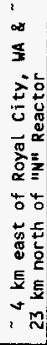 & 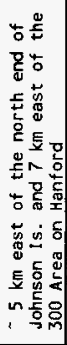 & 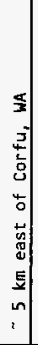 & 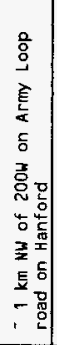 & 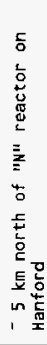 & 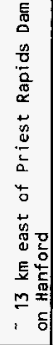 & 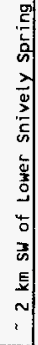 & 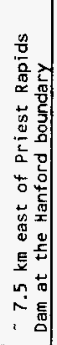 & 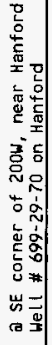 & 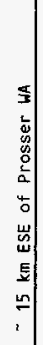 & 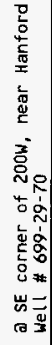 & 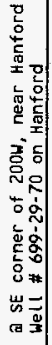 & 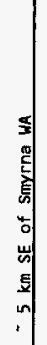 & 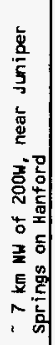 & 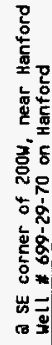 & 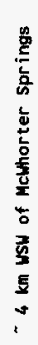 \\
\hline$\infty$ & $\mathbf{m}$ & ن & 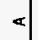 & $<$ & $<$ & $\infty$ & $\infty$ & 0 & $\ll$ & 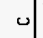 & $m$ & $\infty$ & $m$ & $\infty$ & $\infty$ & m \\
\hline $\begin{array}{l}9 \\
0 \\
0\end{array}$ & $\begin{array}{l}\text { t. } \\
0 \\
0\end{array}$ & $\stackrel{\text { ă }}{\stackrel{0}{0}}$ & m. & 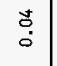 & $\begin{array}{l}m \\
0 \\
0\end{array}$ & $\begin{array}{l}\text { U. } \\
\text { ¿ }\end{array}$ & 包 & 告 & 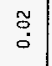 & $\begin{array}{l}\infty \\
0 \\
0\end{array}$ & D. & $\begin{array}{l}\bar{o} \\
0\end{array}$ & $\begin{array}{l}\approx \\
0 \\
0\end{array}$ & $\stackrel{8}{8}$ & $\begin{array}{c}\bar{g} \\
0\end{array}$ & ¿̊. \\
\hline 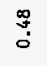 & $\begin{array}{l}\infty \\
\stackrel{\infty}{-} \\
-\end{array}$ & $\stackrel{\stackrel{N}{0}}{\stackrel{0}{0}}$ & $\stackrel{\square}{\circ}$ & $\begin{array}{l}\text { ơ } \\
\stackrel{0}{0}\end{array}$ & $\bar{\sigma}$ & $\bar{m}$ & ב⿳亠口冋. & $\stackrel{\infty}{\stackrel{\infty}{\longrightarrow}}$ & 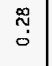 & ஸ़̃ & $\begin{array}{l}\text { ָे } \\
\stackrel{0}{i}\end{array}$ & $\stackrel{8}{8}$ & $\begin{array}{l}\hat{N} \\
0\end{array}$ & $\stackrel{\alpha}{\alpha}$ & :ึ. & 命 \\
\hline$\stackrel{8}{\circ}$ & m. & $\stackrel{\alpha}{\alpha}$ & $\mp$ & $\hat{\tilde{n}}$ & $\stackrel{\alpha}{\sigma}$ & 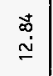 & \begin{tabular}{l}
0 \\
$\infty$ \\
$\infty$ \\
\hdashline
\end{tabular} & $\begin{array}{l}\stackrel{2}{\circ} \\
\stackrel{\circ}{-}\end{array}$ & $\underset{\infty}{\bar{i}}$ & $\begin{array}{l}7 \\
\therefore\end{array}$ & 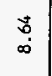 & \begin{tabular}{l}
$\tilde{\infty}$ \\
\multirow{\infty}{*}{}
\end{tabular} & $\stackrel{\tilde{n}}{\tilde{m}}$ & $\stackrel{n}{\circ}$ & $\underset{\infty}{\hat{\infty}}$ & $\frac{\tilde{n}}{\tilde{N}}$ \\
\hline $\begin{array}{l}\bar{\vdots} \\
\frac{\dot{\alpha}}{\sigma}\end{array}$ & 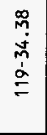 & $\frac{m}{\frac{m}{m}}$ & 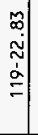 & $\begin{array}{l}\stackrel{9}{9} \\
0 \\
\dot{p} \\
\dot{\alpha} \\
=\end{array}$ & $\begin{array}{l}\hat{v} \\
\dot{m} \\
\dot{\alpha} \\
\underline{=}\end{array}$ & $\begin{array}{l}\stackrel{a}{q} \\
\frac{j}{t} \\
\dot{\alpha} \\
\stackrel{\alpha}{=}\end{array}$ & $\begin{array}{l}0 \\
0 \\
i n \\
\vdots \\
0 \\
=\end{array}$ & $\begin{array}{l}\text { m. } \\
\dot{0} \\
+ \\
\dot{1} \\
0 \\
=\end{array}$ & $\begin{array}{l}\hat{n} \\
\hat{m} \\
\dot{m} \\
\dot{\alpha} \\
\stackrel{0}{0}\end{array}$ & 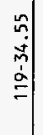 & $\begin{array}{c}\tilde{n} \\
\stackrel{n}{m} \\
\alpha \\
\stackrel{\alpha}{\sigma}\end{array}$ & $\begin{array}{l}\stackrel{P}{0} \\
\stackrel{a}{m} \\
\stackrel{\alpha}{\alpha} \\
\frac{\alpha}{=}\end{array}$ & 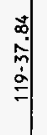 & $\begin{array}{l}R \\
0 \\
o \\
o \\
\stackrel{0}{2} \\
=\end{array}$ & $\begin{array}{l}\text { mे } \\
\text { p. } \\
\stackrel{\alpha}{\underline{\alpha}}\end{array}$ & $\begin{array}{l}2 \\
\stackrel{0}{0} \\
\stackrel{2}{\alpha}\end{array}$ \\
\hline $\begin{array}{c}F \\
\dot{N} \\
\vdots \\
\dot{j}\end{array}$ & 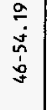 & 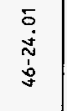 & $\begin{array}{l}5 \\
0 \\
0 \\
0 \\
0 \\
0\end{array}$ & \begin{tabular}{l}
\multirow{2}{*}{} \\
1 \\
$m$ \\
$m$ \\
0 \\
0
\end{tabular} & $\begin{array}{l}9 \\
q \\
\dot{m} \\
+ \\
\vdots \\
0\end{array}$ & $\begin{array}{l}\bar{m} \\
a \\
\tilde{n} \\
\dot{p} \\
\dot{q}\end{array}$ & $\begin{array}{l}0 \\
0 \\
0 \\
1 \\
0 \\
0\end{array}$ & $\begin{array}{l}\alpha \\
\alpha \\
\infty \\
p \\
0 \\
o \\
j\end{array}$ & $\begin{array}{l}\hat{m} \\
\dot{p} \\
\hat{p} \\
\dot{0}\end{array}$ & $\begin{array}{l}8 \\
\dot{0} \\
0 \\
0 \\
0\end{array}$ & $\begin{array}{l}\bar{N} \\
\dot{\rho} \\
\dot{m} \\
\dot{q}\end{array}$ & $\begin{array}{l}\text { Na } \\
\stackrel{0}{0} \\
\dot{b} \\
\dot{6}\end{array}$ & $\begin{array}{l}n \\
0 \\
0 \\
0 \\
0 \\
0\end{array}$ & 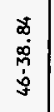 & $\frac{\dot{d}}{\dot{p}}$ & $\begin{array}{l}\bar{a} \\
\grave{̣} \\
\dot{q}\end{array}$ \\
\hline $\begin{array}{l}\stackrel{\infty}{a} \\
\stackrel{0}{0} \\
\ddot{0} \\
\stackrel{0}{0} \\
\ddot{0}\end{array}$ & 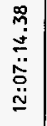 & 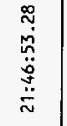 & $\begin{array}{l}N \\
0 \\
0 \\
: \\
\ddot{0} \\
\ddot{0}\end{array}$ & 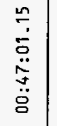 & 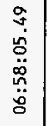 & 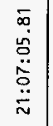 & 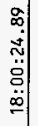 & 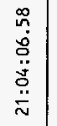 & $\begin{array}{l}\infty \\
\infty \\
\infty \\
\ddot{0} \\
\ddot{N} \\
\ddot{N}\end{array}$ & 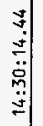 & $\begin{array}{l}\hat{\infty} \\
\stackrel{0}{\circ} \\
\ddot{n} \\
\ddot{0}\end{array}$ & $\begin{array}{l}\tilde{\Xi} \\
\ddot{0} \\
\overline{0} \\
\ddot{0} \\
\ddot{0}\end{array}$ & 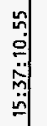 & 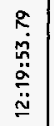 & 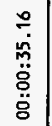 & 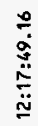 \\
\hline ذे & $\underset{\mathbf{N}}{\mathbf{N}}$ & $\stackrel{\stackrel{m}{2}}{\sim}$ & $\stackrel{\cong}{\cong}$ & $\stackrel{\stackrel{N}{N}}{=}$ & $\stackrel{\Sigma}{\simeq}$ & $\stackrel{ }{\cong}$ & $\stackrel{\infty}{=}$ & $\stackrel{0}{\Xi}$ & $\stackrel{\cong}{\Xi}$ & $\stackrel{\simeq}{\beth}$ & $\stackrel{\Xi}{\risingdotseq}$ & $\stackrel{\cong}{\cong}$ & 휴 & 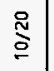 & I̊ & 览 \\
\hline 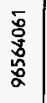 & 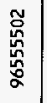 & 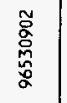 & 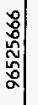 & 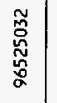 & 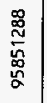 & 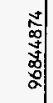 & 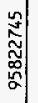 & 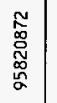 & 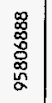 & 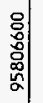 & 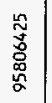 & 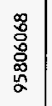 & 告 & 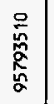 & 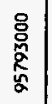 & 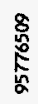 \\
\hline
\end{tabular}


WHC-SD-WM-EV-117, Rev. 0

\subsection{GEOLOGY AND TECTONIC ANALYSIS}

\subsection{DEPTH OF EARTHQUAKES}

Studies of seismicity at the Hanford Site have shown that the seismicity is related to the following crustal stratigraphy (layers of rock types) (DOE 1988).

- The Columbia River Basalt Group (CRBG) (approximately 0-5 km deep)

- The prebasalt sediments (approximately $5-10 \mathrm{~km}$ deep)

- The crystalline basement (greater than approximately $10 \mathrm{~km}$ ).

Since the early 1980s deep boreholes have been drilled for hydrocarbon exploration. These boreholes have provided accurate measurements of the physical properties of the CRBG and the prebasalt sediments (Reidel and others 1987, 1994). The thickness of the prebasalt sediments and nature of the crystalline basement, however, are still poorly understood. The difference between the thickness listed here and the thickness of the crustal layers in the velocity model in Table 4 reflect data specific for the Hanford Site rather than the University of Washington's crustal model for eastern Washington.

Since records have been kept, about 75 percent of the earthquakes at the Hanford site have originated in the CRBG layer. The prebasalt sediments have had about 7 percent of the events and the crystalline basement has had 18 percent.

For fiscal year (FY) 1996, 35 events were located (Table 2); 19 events (54 percent) occurred in the basalt, 6 events (17 percent) in the prebasalt sediments, and 10 events (29 percent) in the crystalline basement.

For the current quarter, 13 events were located (Table 2); 9 events (69 percent) occurred in the basalt, 1 event ( 8 percent) occurred in the prebasalt sediments, and 3 events ( 23 percent) occurred in the crystalline basement.

\subsection{TECTONIC PATTERN}

Studies at the Hanford Site have concluded that earthquakes can occur in the following six different tectonic environments (earthquake sources) at the Hanford Site and in the Columbia Basin of eastern Washington (Geomatrix 1996).

- Reverse thrust faults. Reverse/thrust faults in the CRBG associated with major anticlinal ridges such as Rattlesnake Mountain, Yakima Ridge, and Umtanum Ridge could produce the largest earthquakes. 
WHC-SD-WM-EV-117, Rev. 0

- Secondary faults. These are associated with the major anticlinal ridges.

- Swarm areas. Small geographic areas of unknown geologic structure produce clusters of events (swarms), usually in the CRBG in synclinal valleys. These clusters consist of a series of small shocks with no one outstanding principle event. Swarms occur over a period of days or months and the events may number into the hundreds and then quit, only to start again at a later date. This differs from the sequence of foreshocks, mainshock, and trailingoff aftershocks that have the same epicenter or are associated with the same fault system. Three principal swarm areas are known at the Hanford Site. One is the Wooded Island Swarm Area along the Columbia. River near the 300 Area. The second area, the Coyote Rapids Swarm Area, extends from the vicinity of the $100 \mathrm{~K}$ Area north-northeast along the Columbia River Horn to the vicinity of the $100 \mathrm{~N}$ Area. The third major swarm area is along the Saddle Mountains on the northern boundary of the Hanford Site. Other earthquake swarm areas are present, but activity is less frequent.

- The entire Columbia Basin. The entire basin, including the Hanford Site, could produce a "floating" earthquake. A floating earthquake is one that, for seismic design purposes, can happen anywhere in a tectonic province and is not associated with any known geologic structure. It is classified as a random event by Seismic Monitoring for purposes of seismic design and vibratory ground motion studies.

- Basement source structures. Studies suggest that major earthquakes can originate in tectonic structures in the crystalline basement. Because little is known about geologic structures in the crystalline basement beneath the Hanford Site, earthquakes cannot be directly tied to a mapped fault.

Earthquakes occurring in the crystalline basement without known sources are treated as random events for seismic hazards analysis and seismic design development.

- The Cascadia Subduction Zone. This source recently has been postulated to be capable of producing a 9-CAM earthquake. Because this source is along the western boundary of Washington State and outside the Hanford Array, the Cascadia Subduction Zone is not an earthquake source that is monitared at the Hanford Site, so subduction zone earthquakes are not reported here. Because any earthquake along the Cascadia Subduction zone can have a significant impact on the Hanford Site (Geomatrix 1996), the University of Washington monitors and reports on this earthquake source for the U.S. Department of Energy (DOE). Ground motion from any moderate or larger Cascadia Subduction Zone earthquake is detected by seismometers in the HSN.

\subsection{CURRENT TECTONIC ACTIVITY}

The locations of all events with CAMs greater than 1.0 for FY 1996 are shown on Figure 3. The locations of all seismic events for FY 1996 are shown 
in Figure 4. Figure 5 is a tectonic map of the Yakima Fold Belt and Hanford Site. The Yakima Fold Belt is the main tectonic province of concern for the Hanford Site. The figure shows all the major mapped ridges and faults that are potential seismic sources. These figures should be referred to for the following discussions.

\subsubsection{Reverse/Thrust Faults on Major Anticlinal Ridges}

During this period, one event appears to be associated with one of the major anticlinal ridges. One small (<2.3 CAM) event (96662219) occurred either in the subbasalt sediments or at the base of the basalt under Umtanum Ridge. Projection of the Umtanum Ridge fault zone, including uncertainty of the dip angle of the fault, intersects the hypocenter of this earthquake.

\subsubsection{Secondary Structures on Main Anticlinal Ridges}

During this reporting period, one earthquake (96670943) appears to be associated with possible secondary structures of the Horse Heaven Hills anticlinal ridge. This event occurred on the south flank of the Horse Heaven Hills about $13 \mathrm{~km}$ from the crest of the fold and at a depth of $3 \mathrm{~km}$. It does not appear to be on the main fault, but may be an unmapped secondary structure.

\subsubsection{Swarm Area Activity}

During this reporting period, 6 events (55 percent of a 11 events located) were located in earthquake swarm areas. The swarm areas are discussed in Sections 4.3.3.1 through 4.3.3.4.

4.3.3.1 Saddle Mountains Swarm Area. The Saddle Mountains swarm area experienced one small (<1 CAM) event during the reporting period. This event occurred on April 2, 1996, in the Saddle Mountains at a shallow depth near a known fault that separates two segments of the Saddle Mountains structure. This earthquake might be fault controlled.

4.3.3.2 Coyote Rapids Swarm Area. One earthquake occurred in the Coyote Rapids swarm on May 26, 1996. This was a sma11-magnitude earthquake (<1 CAM) at the north end of the swarm area west of $100 \mathrm{D}$ Area.

4.3.3.3 Wooded Island Swarm Area. Six events occurred here during the reporting period. Four events occurred between May 15 and May 24, 1996. These events occurred near Johnson Island, one near the north end of the swarm and three a few kilometers east of the island. The sixth event occurred on April 18, 1996, near the southwest end of Wooded Island. A1l events were less than 2.2 CAM.

4.3.3.4 0ther Swarm Areas. No other swarm areas were active during this reporting period. 
Figure 3. All Located Earthquakes: April 1, 1996, and June 30, 1996. (Coda Amplitude Scale is shown at the top of the Map.)

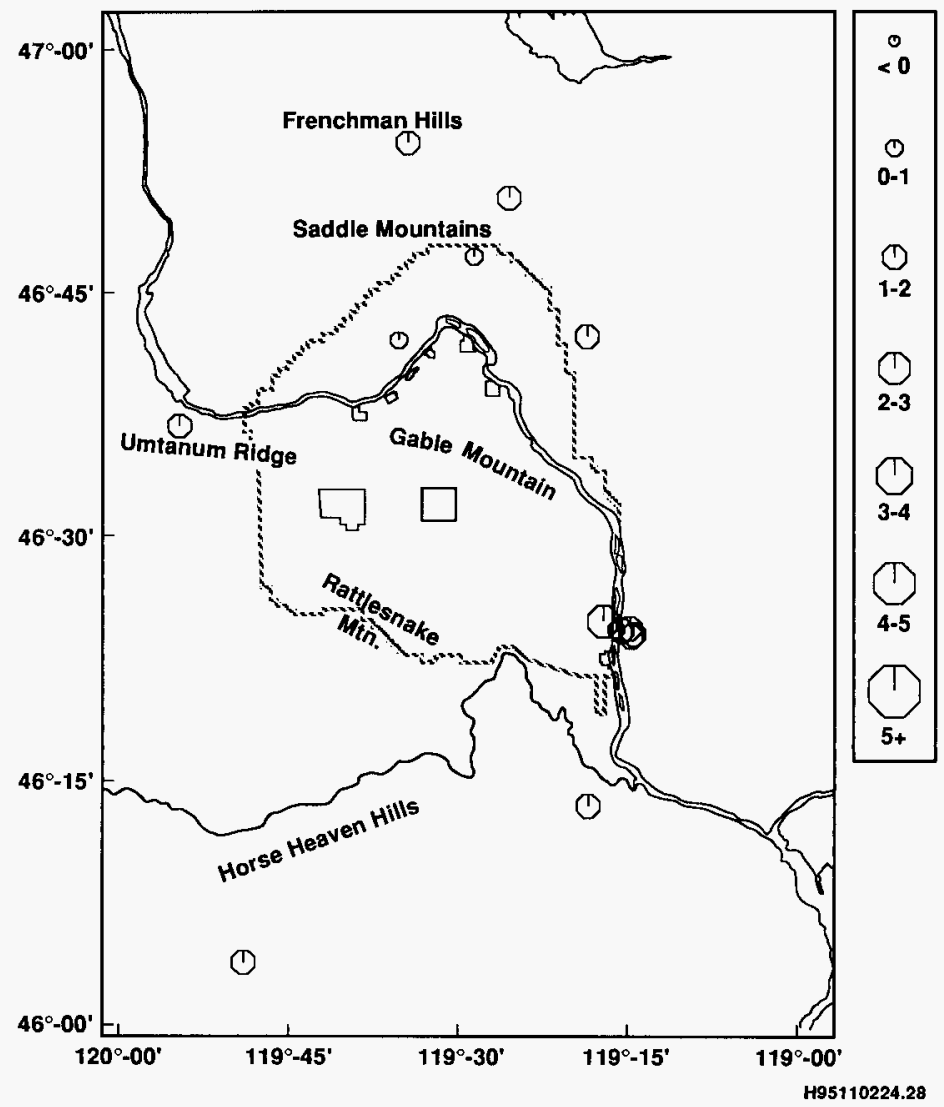


Figure 4. Locations of A11 FY 1996 Events: (Coda Amplitude Magnitude Scale is shown at the top of the map.)

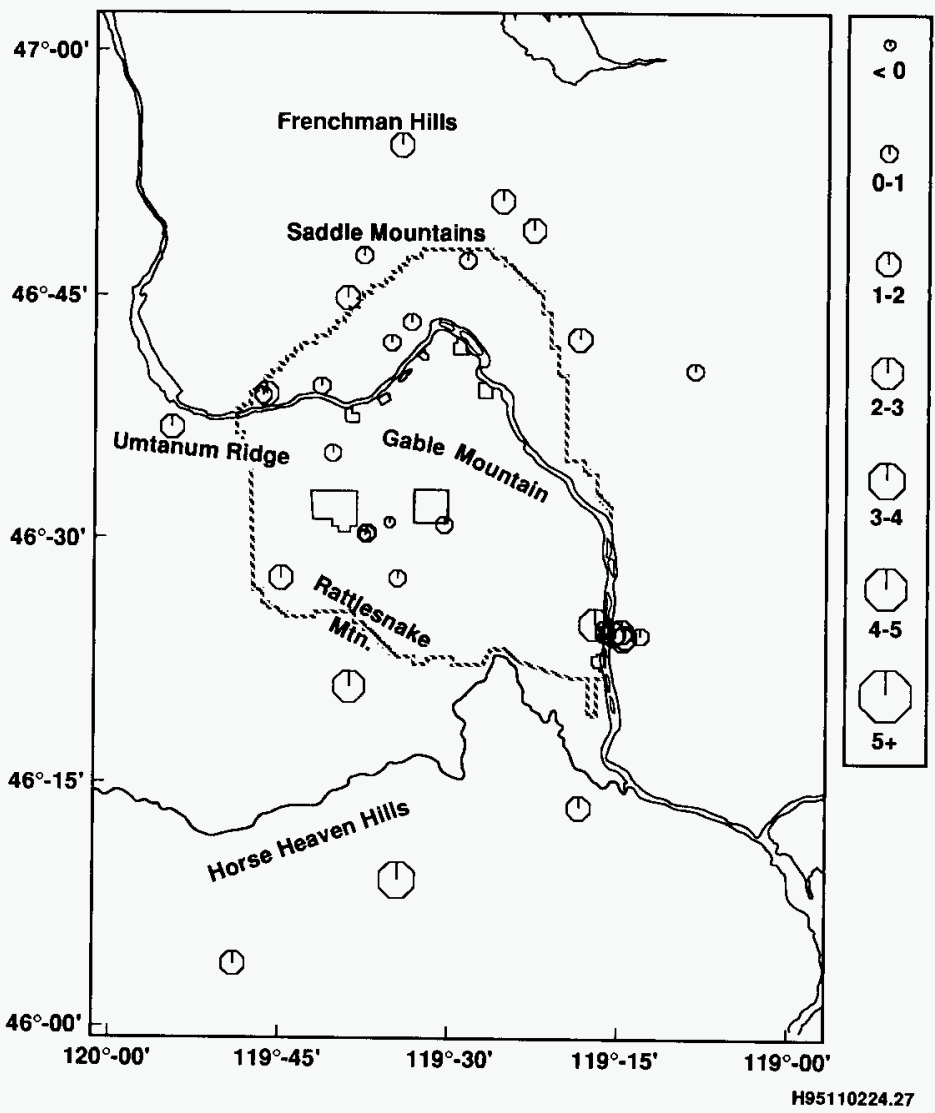


Figure 5. Structural and Tectonic Map of Columbia Basin Showing Major Seismic Source Structures.

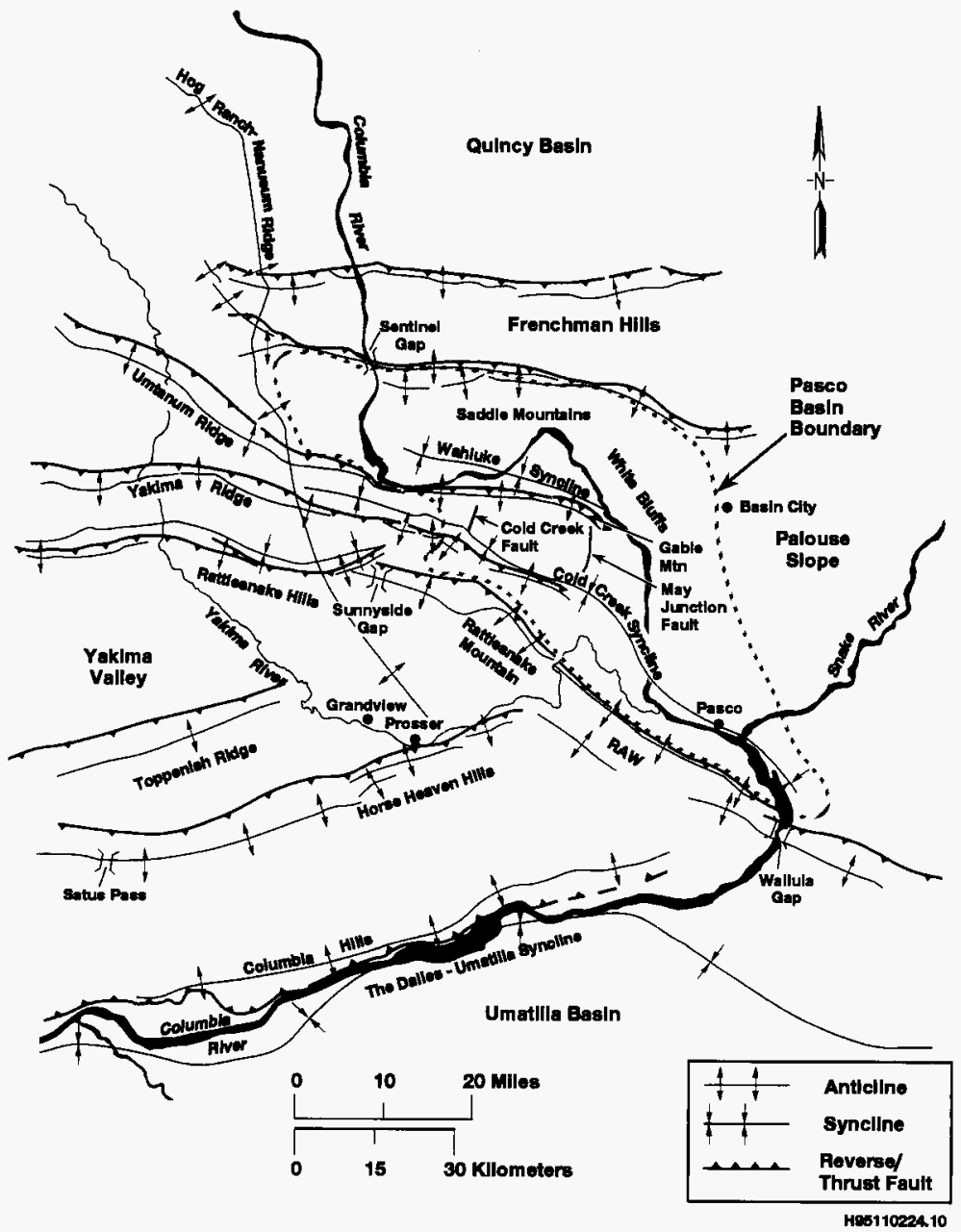




\subsubsection{Random or Floating Event Areas}

Four events that occurred during the reporting period ( 36 percent of all events located) are classified as random events. The areas are discussed in Sections 4.3.4.1 through 4.3.4.6.

4.3.4.1 Saddle Mountains Area. On May 9, 1996, a smal1 (<2 CAM) event occurred at a depth of $17.1 \mathrm{~km}$ in the crystalline basement on the north flank of the Saddle Mountains. This is classified as a random event because no known fault occurs at this depth. This earthquake occurred in the Saddle Mountains earthquake swarm area, but could have been along the Frenchman Hills fault zone. Because the earthquake occurred below the basalt in the crystalline basement and studies of the Frenchman Hills fault suggest that it does not extend below the basalt, this event is presentiy not considered to be associated with that fault.

A second event ( $<1.5$ CAM) occurred $8 \mathrm{~km}$ southwest of the crest of the Saddle Mountains anticline at a depth of $27.8 \mathrm{~km}$. This earthquake occurred on the south flank of the Saddle Mountains, but could have been along the Saddle Mountains fault zone in the crystalline basement. Because the earthquake occurred below the basalt in the crystalline basement and studies of the Saddle Mountains fault suggest that it does not extend below the basalt, this event is presently not considered to be associated with that fault. 


\subsection{REFERENCES}

Geomatrix, 1996, Probabilistic Seismic Hazard Analysis, DOE Hanford Site, Washington, WHC-SD-W236A-TI-002, Rev. 1, Westinghouse Hanford Company, Richland, Washington.

Reide1, S. P., N. P. Campbe11, K. R. Fecht, and K. A. Lindsey, 1994, "Late Cenozoic Structure and Stratigraphy of South-Central Washington," in Cheney, E. and R. Lasmanis, Eds, Regional Geology of Washington State, Washington Division of Geology and Earth Resources Bulletin 80, pp 159-180, 0lympia, Washington.

Reide1, S. P., K. R. Fecht, M. C. Hagood, and T. L. Tolan, 1989, "Geologic Development of the Central Columbia Plateau," in Reidel, S.P. and P. R. Hooper, Eds., Volcanism and Tectonism in the Columbia River Flood-Basalt Province Geological Society of America Special Paper 239, pp. 247-264.

DOE, 1988, Site Characterization Plan for the Reference Location, Hanford, Washington-Consultation Draft, Report D0E/RW-0164, Vol. 1, U.S. Department of Energy, Washington, D.C. 
WHC-SD-WM-EV-117, Rev. 0

DISTRIBUTION

Number of Copies

Onsite

2

U.S. Department of Energy. Richland Operations office

M. J. Furman

H4-85

J. E. Mecca

R3-79

K. M. Thompson

$\mathrm{H} 4-83$

1 Bechtel Hanford, Inc. P. J. Mackey B3-15

36 Westinghouse Hanford Company

M. V. Berriochoa A3-01

J. A. Caggiano H6-06

D. A. Conners T5-11

A. J. Diliberto (2) H6-10

J. W. Fassett H6-06

J. C. Fulton R3-11

F. T. Green H6-29

D. C. Hartshorn (15) H6-06

J. E. Mecca R3-79

R. A. Meznarich H6-30

D. L. Mitchell H6-29

S. R. Moreno B3-06

S. P. Reidel H6-06

E. R. Siciliano H0-31

A. M. Tallman H6-30

R. R. Thompson R2-12

J. D. Williams H6-30

B. D. Wiltiamson B3-15

M. I. Wood T3-01

M. T. York H6-29

Central Files A3-88 
WHC-SD-WM-EV-117, Rev. 0

This page intentionally left blank.

Distr-2 


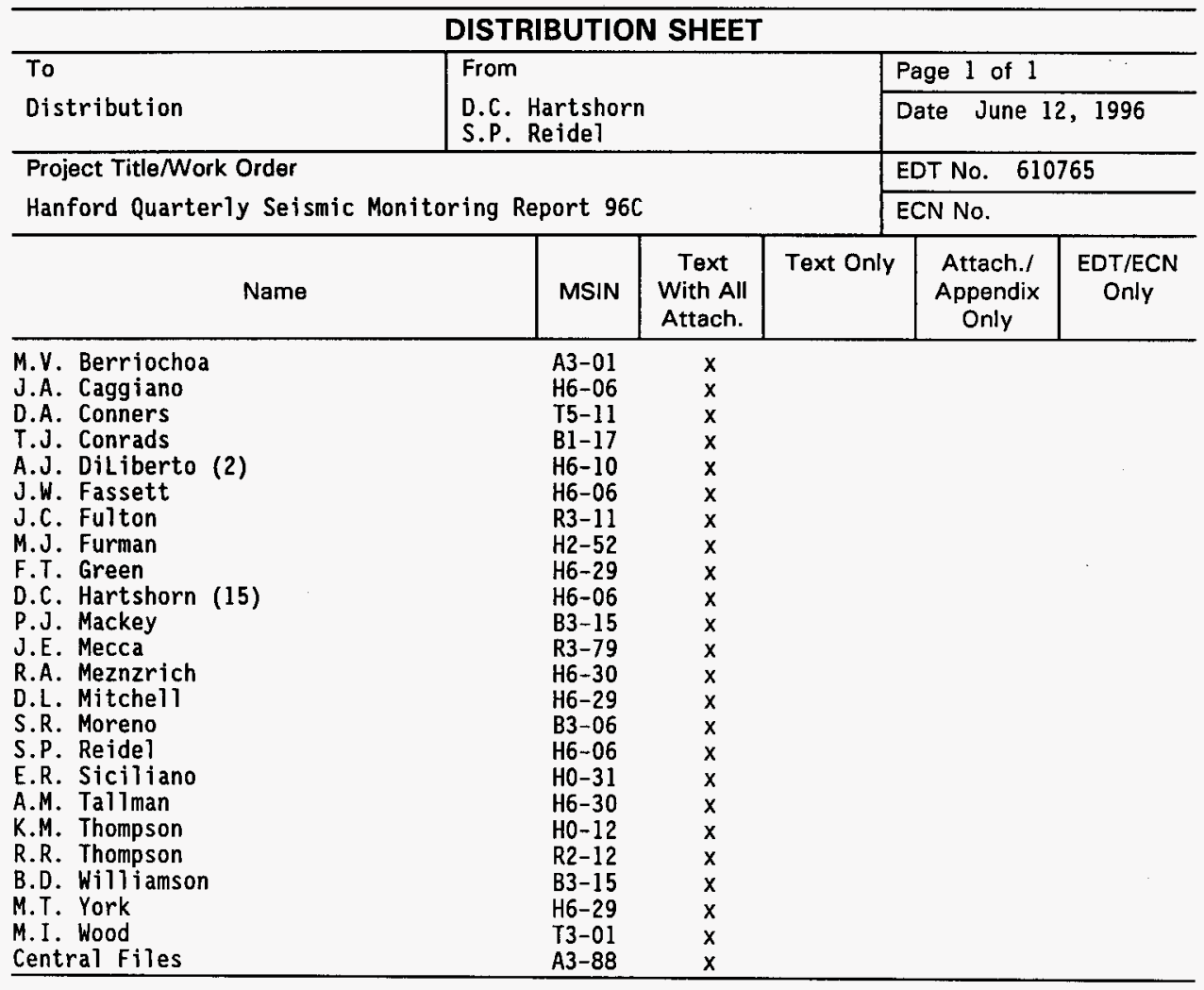

